\title{
Human hepatocyte growth factor inhibits early neointima formation in rabbit abdominal aortae following ultrasound-guided balloon injury
}

\author{
LI MEI $^{1,2^{*}}$, YU HE ${ }^{1 *}$, HUI WANG ${ }^{1}$, YING JIN ${ }^{3}$, SHUAI WANG ${ }^{4}$ and CHUNXIANG JIN ${ }^{1}$ \\ ${ }^{1}$ Department of Ultrasound, China-Japan Union Hospital of Jilin University, Changchun, Jilin 130033; \\ Departments of ${ }^{2}$ Ultrasound and ${ }^{3}$ Surgery, The First Hospital of Jilin University, Changchun, Jilin 130021; \\ ${ }^{4}$ Department of Pathology, Cancer Hospital of Jilin, Changchun, Jilin 130012, P.R. China
}

Received October 24, 2016; Accepted June 8, 2017

DOI: $10.3892 / \mathrm{mmr} .2017 .7229$

\begin{abstract}
The present study investigated the effects of in vivo gene transfer of human hepatocyte growth factor (hHGF) on neointima formation in rabbit abdominal aortae following ultrasound-guided balloon injury. New Zealand white rabbits were randomly divided into four groups: endothelium injury alone (EI), endothelium injury with control vector transfection (EI-V), endothelium injury with hHGF transfection (EI-HGF), and hHGF transfection alone without endothelium injury (HGF). Endothelial injury was established by scraping the abdominal aortic wall using a balloon catheter under the guidance of a transabdominal ultrasound. hHGF gene transfer was performed 7 days following injury. hHGF mRNA and protein expression levels were determined at 3, 7, 14 and 21 days following transfection. Neointima formation was assessed by histopathological analysis at 14 and 28 days following injury. hHGF mRNA and protein expression levels were detected in the target abdominal aortae in EI-HGF and HGF groups with the greatest levels observed 3 days following transfection, and their levels dropped below detection limits at 21 days following transfection. hHGF was not detectable in the EI and EI-V groups throughout the experiment. The neointimal area and the neointima to media ratio in the EI-HGF group were significantly decreased compared with those in the EI or EI-V group at 14 days following injury. However, no differences were observed at 28 days following injury. The present
\end{abstract}

Correspondence to: Professor Chunxiang Jin, Department of Ultrasound, China-Japan Union Hospital of Jilin University, 126 Xiantai Street, Changchun, Jilin 130033, P.R. China

E-mail: jinchunxiang0115@163.com

*Contributed equally

Abbreviations: HGF, Human hepatocyte growth factor; VSMC, vascular smooth muscle cells

Key words: hepatocyte growth factor, neointima formation, gene therapy, vascular injury, ultrasound-guided study demonstrated that in vivo hHGF gene transfer inhibits the early formation of neointima in balloon-injured rabbit abdominal aortae.

\section{Introduction}

Endovascular procedures, including balloon angioplasty, are frequently used to widen or unblock coronary or peripheral arteries to increase blood flow. However, these procedures damage the artery walls, and the vessels respond by activating physiological mechanisms to repair the damage. This repair process primarily involves vascular smooth muscle cell (VSMC) proliferation, migration and production of extracellular matrix, leading to neointimal hyperplasia and re-narrowing of arterial walls; an adverse event called restenosis (1). Endothelial cells (ECs) lining the inner surface of the vessel wall secrete a number of factors that suppress VSMC growth and migration, including prostaglandin, nitric oxide and heparan sulfate $(2,3)$. Therefore, agents that promote re-endothelialization following vascular injury would likely inhibit VSMC proliferation and migration, thereby preventing post-surgery neointimal hyperplasia. Indeed, vascular endothelial growth factor, a mitogenic factor exclusive to ECs, has been demonstrated to accelerate re-endothelialization and attenuate neointima formation following arterial wall injury (4-6).

Hepatocyte growth factor (HGF) is a pleiotropic factor that regulates cell growth, cell motility and morphogenesis by binding to and activating the proto-oncogenic tyrosine-protein kinase Met (c-MET) receptor (7,8). HGF is primarily secreted by mesenchymal cells and acts primarily on epithelial cells and ECs. It serves a major role in embryonic development and in adult organ regeneration and wound healing (9-12). HGF is also expressed in a number of other cell types, including ECs and smooth muscle cells (SMCs) $(12,13)$. Importantly, studies have revealed that $\mathrm{HGF}$ stimulates growth of ECs exclusively; however, it does not induce SMCs $(14,15)$. In addition, administration of recombinant HGF, or gene transfer of HGF, inhibits neointima formation via rapid re-endothelialization following invasive endovascular procedures $(16,17)$. However, little is known about the time course and efficiency of HGF 
expression following in vivo gene transfer. In the present study, the effects of in vivo human HGF (hHGF) transfer on neointima formation in balloon-injured rabbit abdominal aortae were investigated. Real-time ultrasound monitoring was applied in the balloon-injury procedure to keep injury degree well-controlled. The association between neointima formation and the time course and efficiency of HGF expression was investigated.

\section{Materials and methods}

Animals. New Zealand white rabbits ( $\mathrm{n}=86$; males and females; age, 4-6 months; weighing 2.0-2.5 kg) were purchased from the Experimental Animal Center of Jilin University (Changchun, China). Rabbits were housed in isolated cages under constant conditions (18-25 $\mathrm{C}$; 40-60\% humidity; 12-h light/dark cycle) with free access to food and water for one week prior to experiments. All animal experiments were performed following the Guide for the Care and Use of Laboratory Animals of the National Institute of Health (18). This study was approved by the Animal Care and Use Committee of China-Japan Union Hospital of Jilin University (Jilin, China).

Abdominal aortic balloon-injury model. All surgeries were performed under adequate anesthesia with sodium pentobarbital $(30 \mathrm{mg} / \mathrm{kg})$ via the ear vein. Eighty-six rabbits were randomly allocated into four groups: Endothelium injury alone $(E I, n=22)$, endothelium injury with control vector transfer (EI-V, $n=22)$, endothelium injury with hHGF transfer (EI-HGF, n=30) and hHGF transfer alone without endothelium injury (HGF, $n=12$ ). The experimental protocol is depicted in Fig. 1.

Endothelial injury was established by scraping the abdominal aortic wall using a balloon catheter, which was monitored by a high-frequency duplex ultrasonographic system (Esaote, Genoa, Italy) with a $7.5 \mathrm{MHz}$ transducer. Prior to the procedure, transabdominal ultrasonography was performed; a middle segment of the abdominal aorta (AA, $2 \mathrm{~cm}$ in length, 3-6 cm below the left renal vein) was selected as the target site. Then, a balloon catheter of 3.0-3.5 mm (Boston Scientific Corporation, Natick, MA, USA) was introduced into the aorta via the exposed femoral artery under the guidance of ultrasonography (Fig. 2A). Color Doppler ultrasound demonstrated normal blood flow around the balloon catheter (Fig. 2B). Following catheter advancement to the level of the renal artery, the balloon was inflated with normal saline guided by ultrasound. The blood flow disappeared gradually until no flow signal was detected, indicating aortic occlusion by the inflated balloon (Fig. 2C and D). The balloon was inflated continuously until resistance was felt when the catheter was pulled along the vessel. Then, the anteroposterior diameter of the inflated balloon was measured. The ratio of the inflated balloon diameter to that of the aortic artery was $1: 2$, in accordance with previous studies $(19,20)$. The catheter was subsequently retracted toward the iliac bifurcation, and this was repeated three times to ensure complete endothelial denudation. The balloon catheter was then removed, and the femoral artery was ligated using a 4-0 silk braided non-absorbable suture. The ratio of inflated balloon to artery was kept $\sim 1: 2$ in all subsequent experiments to ensure the same degree of injury.
In vivo gene transfer. A total of 7 days following balloon injury, rabbits were transfected with hHGF plasmids (HGF and EI-HGF groups) or vector alone (EI-V group), as previously described (16). Briefly, the abdomen was opened with a median incision and the bowels were pushed aside to expose the abdominal aorta. The target segment was dissociated appropriately and occluded at both ends using artery clamps. Residual blood was extracted, and $0.1 \mathrm{ml}$ of the NL003-PCK plasmid containing $0.2 \mathrm{mg}$ of the hHGF insert or the control vector (both were provided by Beijing Northland Biotech Co., Ltd., Beijing, China; stored at $-20^{\circ} \mathrm{C}$ prior to use) was infused by a cannula. Following incubation for $10 \mathrm{~min}$ at room temperature, the cannula was removed immediately and a sterile gauze was applied on the pinhole for compression hemostasis. Subsequently, the clamps were released, and blood flow was restored. The transfection site was marked with suture on the posterior peritoneum. Finally, the surgical incision was subsequently sutured. Rabbits in the HGF group were transfected with hHGF plasmid following the aforementioned procedure, without prior endothelial injury.

Aorta harvest and preparation. On days 10, 14, 21 and 28 following balloon injury (days 3, 7, 14 and 21 following transfection), the target aortae segment in each group was dissected while the animals were under deep anesthesia, and the animals were sacrificed immediately following the dissection. A total of 3-5 rabbits per group were used at each time point for hHGF detection. Surrounding tissues were carefully removed from the aorta segments. Following washing with physiological saline solution, each segment was divided into two parts (for measurement of hHGF mRNA and protein expression, respectively) and frozen in liquid nitrogen. For pathohistological analysis, the target segments in the EI, EI-V and EI-HGF groups were isolated on days 14 and 28 following balloon injury, fixed in $10 \%$ neutralized formalin for $24 \mathrm{~h}$ at room temperature, and embedded in paraffin. Cross-sections (4 $\mu \mathrm{m})$ were prepared and stained with hematoxylin-eosin (H\&E; hematoxylin staining for $5 \mathrm{~min}$ and eosin staining for $2 \mathrm{~min}$ ) at room temperature. A total of 5 rabbits per group were used at each time point for morphological evaluation.

Assessment of hHGF mRNA expression by semi-quantitative polymerase chain reaction ( $s q P C R$ ). Frozen tissues were weighed, placed in a mortar containing liquid nitrogen and homogenized with a grinding rod. Total RNA was isolated using TRIzol ${ }^{\circledR}$ reagent (Thermo Fisher Scientific, Inc., Waltham, MA, USA), as previously described (21). RNA concentrations and purities were determined on a NanoDrop 2000 spectrophotometer (Thermo Fisher Scientific, Inc.). Total RNA concentrations were calculated in the range of 500-1,200 $\mathrm{ng} / \mu \mathrm{l}$ based on absorbance (A) 260/280 ratios of 1.8-2.0. Complementary DNA (cDNA) was synthesized from $1 \mu \mathrm{g}$ RNA using the PrimeScript ${ }^{\mathrm{TM}}$ II 1st strand cDNA Synthesis kit (Takara Bio, Inc., Otsu, Japan) on a MyCycler (Bio-Rad Laboratories, Inc., Hercules, CA, USA). PCR was performed using $2 \mu \mathrm{l}$ of cDNA and $12.5 \mu \mathrm{l}$ of 2X Premix Taq ${ }^{\mathrm{TM}}$ (Takara Bio, Inc.) on a MyCycler as follows: An initial predenaturation step at $98^{\circ} \mathrm{C}$ for $1 \mathrm{~min}$, followed by 40 cycles of denaturation at $98^{\circ} \mathrm{C}$ for $10 \mathrm{sec}$, annealing at $62^{\circ} \mathrm{C}$ for $30 \mathrm{sec}$, extension at $72^{\circ} \mathrm{C}$ for $48 \mathrm{sec}$ and a final extension for 5 min at $72^{\circ} \mathrm{C}$. A total of 40 cycles were performed in the present 


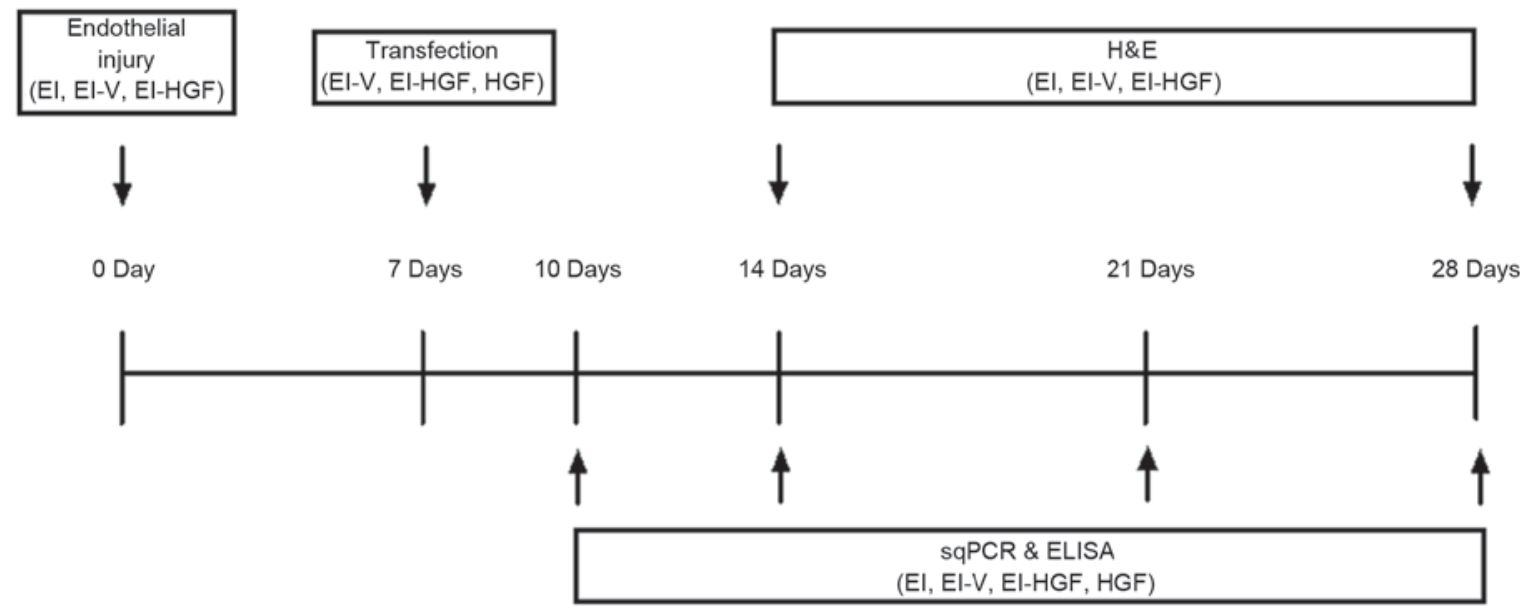

Figure 1. Schematic diagram of study design. HGF, uninjured aorta transfected with human hepatocyte growth factor; EI-HGF, injured aorta transfected with human hepatocyte growth factor; EI, injured aorta without transfection; EI-V, injured aorta transfected with vector alone; H\&E, hematoxylin-eosin; sqPCR, semi-quantitative polymerase chain reaction.
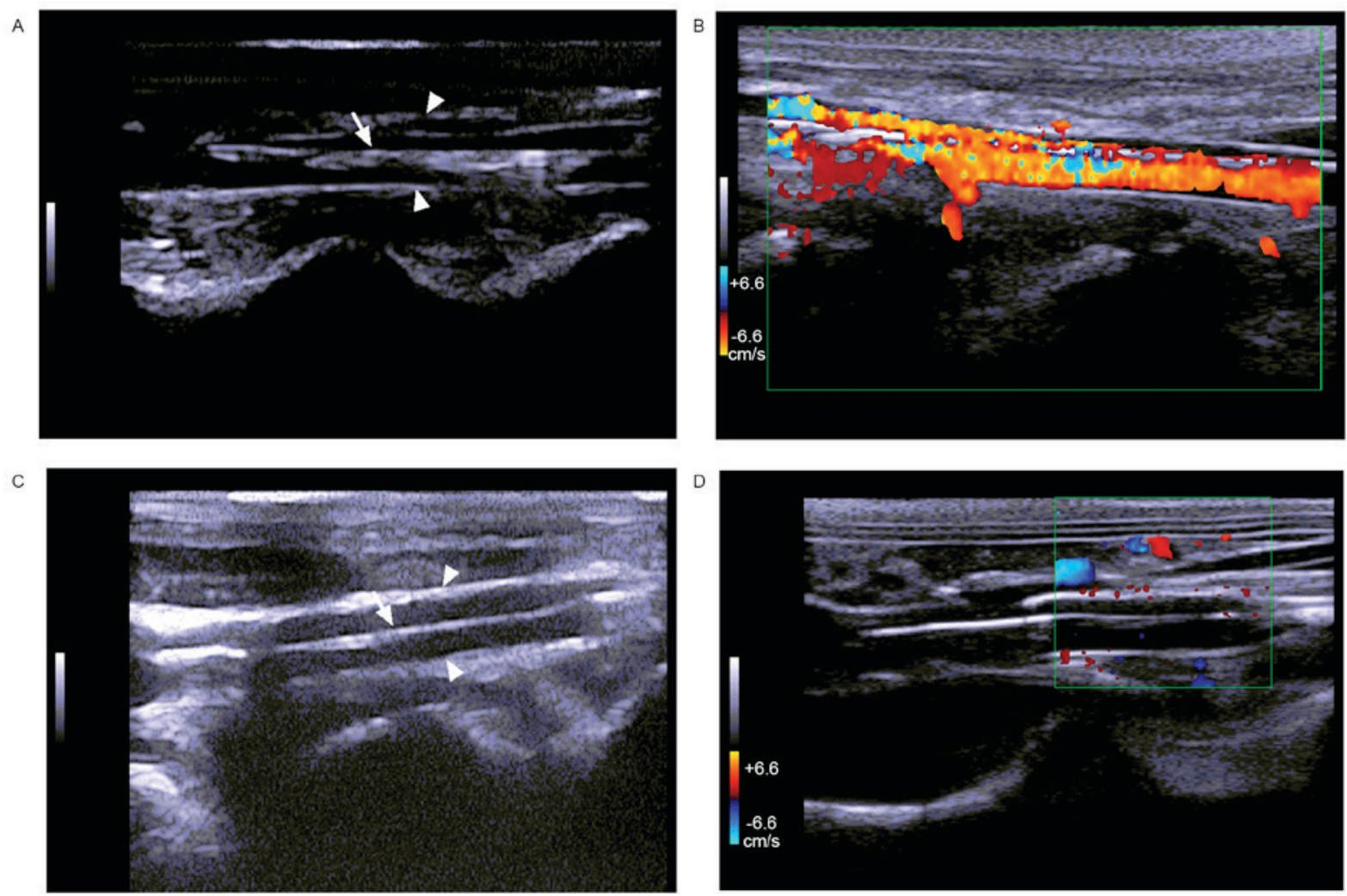

Figure 2. The ultrasound images of balloon injury procedure in rabbit abdominal aortae. (A) Deflated balloon in abdominal aorta demonstrated as a hyperechoic long line. (B) Normal blood flowaround the deflated balloon by color Doppler ultrasound imaging. (C) Inflated balloon pressing against the vessel wall. The line at the center of the vessel indicated the catheter guidewire. (D) Loss of blood flow around the inflated balloon by color Doppler ultrasound imaging. The red coloring indicates normal blood flow while the blue coloring outside of the target segment represents small collateral vessels around the aorta. Arrow heads indicate the wall of abdominal aortae. Arrows indicate the balloon.

study as bands were not observed at lower cycle numbers. $\beta$-actin served as an internal control. The primers specific for hHGF were 5'-ATGTGGGTGACCAAACTCCT-3' (forward) and 5'-TCATCAAAGCCCTTGTCGG-3' (reverse), which amplify a 772-bp product. The primers for $\beta$-actin were 5'-TCTTCCAGC CCTCCTTCCTG-3' (forward) and 5'-CGTTTCTGCGCCGTT AGGT-3' (reverse), which amplify a 409-bp product. The PCR products were electrophoretically separated on $1.5 \%$ agarose gels containing $0.1 \%$ ethidium bromide and detected on a Gel Doc $^{\mathrm{TM}}$ XR+ Imaging System (Bio-Rad Laboratories, Inc.) under ultraviolet fluorescent light. Image Lab software (version 4.0; Bio-Rad Laboratories, Inc.) was used for densitometry. Data was normalized to $\beta$-actin. Each sample was tested in triplicate.

Assessment of hHGF protein expression by ELISA assay. The frozen tissues were weighed and homogenized using Tissue 
Extraction Reagent I (Invitrogen; Thermo Fisher Scientific, Inc.) with a protease inhibitor cocktail (Sigma-Aldrich; Merck KGaA, Darmstadt, Germany). The homogenates were centrifuged at $9,600 \times \mathrm{g}$ at $4^{\circ} \mathrm{C}$ for $5 \mathrm{~min}$. The supernatants were collected. The concentration of hHGF was determined using a Quantikine ELISA Human HGF kit (cat. no. DHG00; R\&D Systems, Inc., Minneapolis, MN, USA) following the manufacturer's protocol. Each sample was tested in triplicate.

Pathohistological aortic analysis. The H\&E stained tissue sections from the EI, EI-V and EI-HGF groups were examined under an Olympus DP73 microscope (Olympus Corporation, Tokyo, Japan). The digital images were analyzed using Image-Pro Plus software, version 6.0 (Media Cybernetics, Inc., Rockville, MD, USA). Neointimal area (NA), defined as the area between the lumen and the internal elastic lamina, was calculated in each animal as the mean of three measurements at different sites of the target segment. Medial area (MA) was defined as the area between the internal and external elastic laminae. The ratio of NA to MA (N/M ratio) was calculated. The samples were examined at the Cancer Hospital of Jilin Province (Jilin, China) by two histologists who were blinded to the treatment conditions.

Statistical analysis. Results are presented as the mean \pm standard error. Differences between groups were analyzed using an analysis of variance and Bonferroni post hoc test. $\mathrm{P}<0.05$ was considered to indicate a statistically significant difference. Statistical charts were prepared using GraphPad Prism software, version 5.0 (GraphPad Software, Inc., La Jolla, CA, USA).

\section{Results}

Consistency in abdominal aortae balloon injury under ultrasound monitoring. The diameters of AA and the inflated balloon in the EI, EI-V and EI-HGF groups are demonstrated in Table I. No statistical significant differences were observed among the EI, EI-V and EI-HGF groups $(\mathrm{P}>0.05)$. The degree of injury was well controlled with ultrasound monitoring.

hHGF mRNA expression following in vivo hHGF gene transfection in rabbit abdominal aortae. hHGF mRNA expression in rabbit abdominal aortae was measured 3, 7, 14 and 21 days following transfection using sqPCR. hHGF mRNA expression was not detected in the EI or EI-V groups throughout the experiment (Fig. 3A); however, it was detected in the EI-HGF and HGF groups at 3, 7, and 14 days following transfection (10, 14, and 21 days following injury; Fig. 3A and B). In addition, hHGF mRNA expression peaked at 3 days following transfection in injured and uninjured aortae, and gradually declined over time $(\mathrm{P}<0.05$ vs. 3 days post-transfection within groups) until the hHGF levels dropped below the detection limit at 21 days post-transfection (28 days following injury; Fig. 3A and B). One possible explanation for the decrease in hHGF expression could be the gradual degradation of transfected exogenous hHGF plasmid over time. Incompatibility between species (human and rabbit) may also contribute to this outcome. Compared with the HGF group, hHGF mRNA expression levels in aortae of the EI-HGF group
Table I. Comparison of parameters of balloon injury among groups.

\begin{tabular}{lccc}
\hline & $\begin{array}{c}\text { AA diameter } \\
(\mathrm{mm})\end{array}$ & $\begin{array}{c}\text { Inflated balloon } \\
\text { diameter }(\mathrm{mm})\end{array}$ & $\begin{array}{c}\text { Ratio } \\
\text { (balloon/AA) }\end{array}$ \\
\hline EI & $2.671 \pm 0.034$ & $3.229 \pm 0.040$ & $1.209 \pm 0.004$ \\
EI-V & $2.663 \pm 0.034$ & $3.245 \pm 0.052$ & $1.220 \pm 0.019$ \\
EI-HGF & $2.689 \pm 0.030$ & $3.243 \pm 0.039$ & $1.206 \pm 0.003$ \\
\hline
\end{tabular}

Data are expressed as the mean \pm standard error. AA, abdominal aorta; EI, injured aorta without transfection; EI-V, injured aorta transfected with vector alone; EI-HGF, injured aorta transfected with human hepatocyte growth factor.

was significantly lower at 3 days post-transfection $(\mathrm{P}<0.05$; Fig. 3B). However, no significant differences were detected between the two groups at 7 or 14 days post-transfection (P>0.05; Fig. 3B). Decreased mRNA expression levels of hHGF in injured aortae 3 days following transfection may have resulted from impaired transfection and gene expression capacity of the injured tissue.

hHGF protein expression following in vivo hHGF gene transfection in rabbit abdominal aortae. hHGF protein expression in rabbit abdominal aortae was detected by an ELISA kit specific for hHGF. In this kit, a specific anti-hHGF antibody that did not cross-react with rabbit HGF was used to detect the hHGF protein. Similar to the PCR results, the ELISA did not detect the hHGF protein in the EI or EI-V throughout the experiment (data not shown). The hHGF protein, however, was detected in the EI-HGF and HGF groups at 3, 7 and 14 days post-transfection, with peak levels detected following 3 days, followed by a gradual decline until the levels dropped below the detection limit at 21 days following transfection (Fig. 4). hHGF protein expression in the EI-HGF group was significantly lower compared with the HGF group at 3 days post-transfection $(\mathrm{P}<0.05)$. hHGF mRNA levels in the EI-HGF group were similar to those in the HGF group at 7 and 14 days post-transfection; however, the protein expression levels in the EI-HGF group were still significantly lower at these time points $(\mathrm{P}<0.05$, Fig. 4). These results may suggest that, in injured aortic tissues, the mRNA expression machinery recovered more quickly than protein expression following the insult.

Mitigation of early neointima formation following in vivo hHGF gene transfection in injured rabbit abdominal aortae. Neointima formation in rabbit abdominal aortae was assessed following 14 and 28 days injury ( 7 and 21 days following the transfection) by pathohistological analysis with H\&E staining. As demonstrated in Fig. 5A, neointima was detected at the target site 14 days following injury in the EI, EI-V and EI-HGF groups. Whereas the MA remained stable, the NA in these three groups increased from 14 to 28 days following injury (Fig. 5B). At a total of 14 days following injury, the NA and $\mathrm{N} / \mathrm{M}$ ratio in the EI-HGF group were significantly decreased compared with those in the EI-V or EI groups $(\mathrm{P}<0.05$; Fig. 5B). These results suggested that hHGF transfection had a 
A

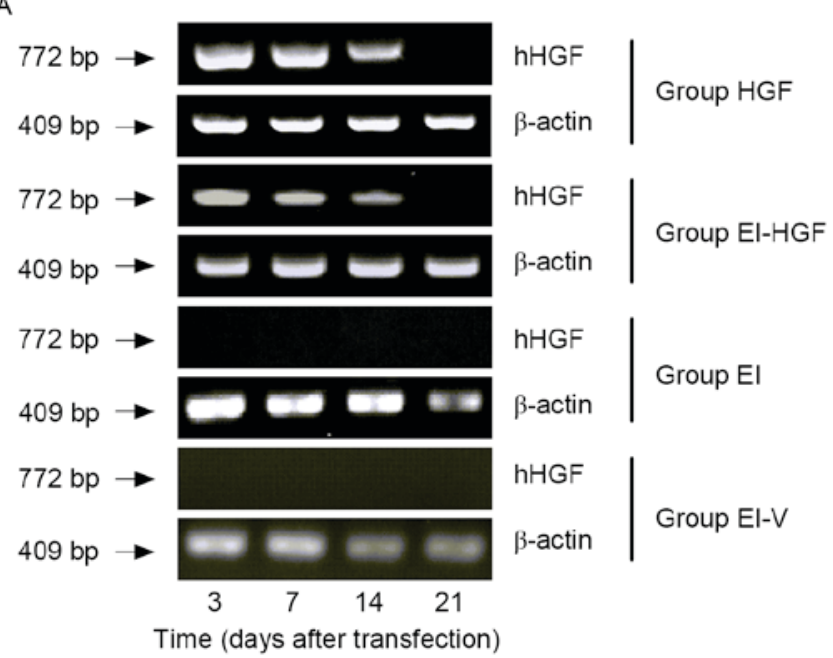

B

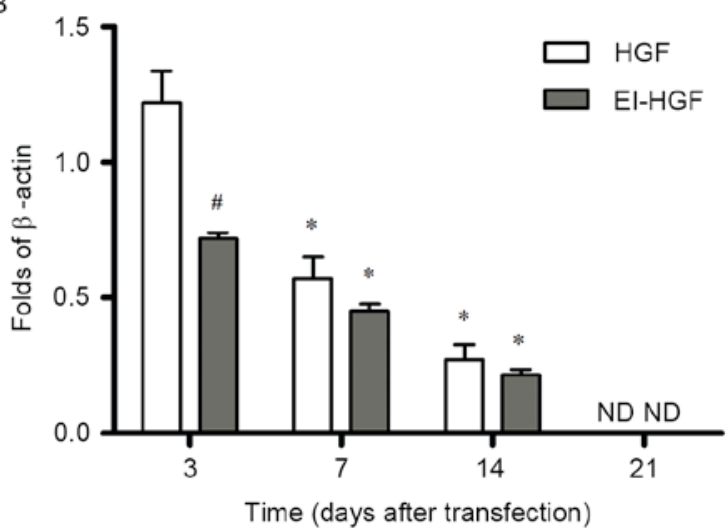

Figure 3. hHGF mRNA expression levels at 3, 7, 14 and 21 days following in vivo hHGF transfection using semi-quantitative polymerase chain reaction. (A) Agarose gel electrophoresis of PCR products. (B) hHGF mRNA expression levels in the HGF and EI-HGF groups (expressed as fold-change relative to $\beta$-actin). Values are presented as the mean \pm standard error. ${ }^{*} \mathrm{P}<0.05$ vs. data at 3 days following transfection within groups; ${ }^{*} \mathrm{P}<0.05$ vs. HGF on the same day. HGF, uninjured aorta transfected with human hepatocyte growth factor; EI-HGF, injured aorta transfected with human hepatocyte growth factor; EI, injured aorta without transfection; EI-V, injured aorta transfected with vector alone; ND, not detected.

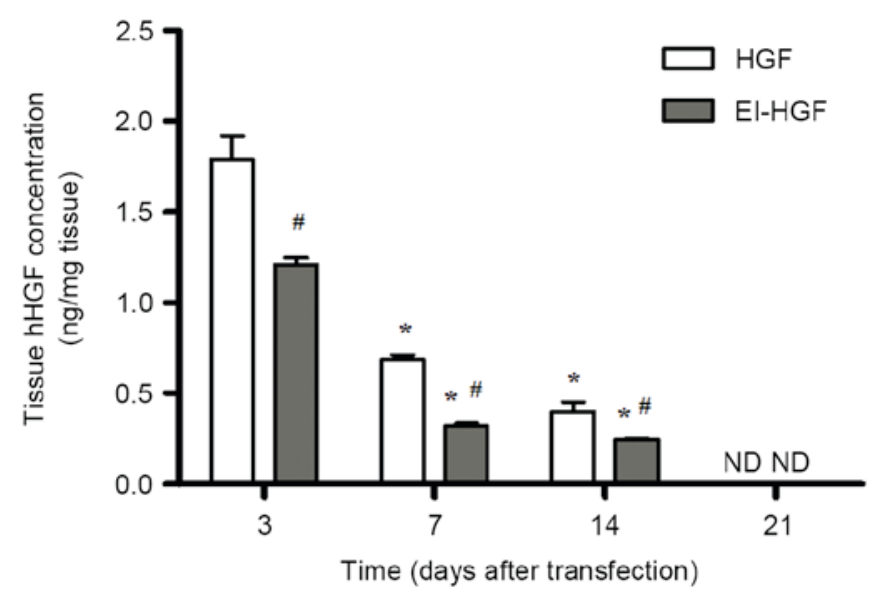

Figure 4. hHGF protein expression at 3, 7, 14 and 21 days following in vivo hHGF transfection using ELISA. Values are presented as the mean \pm standard error. ${ }^{*} \mathrm{P}<0.05$ vs. data at 3 days post-transfection within groups; ${ }^{\#} \mathrm{P}<0.05$ vs. HGF on the same day. HGF, uninjured aorta transfected with human hepatocyte growth factor; EI-HGF, injured aorta transfected with human hepatocyte growth factor; ND, not detected. protective effect against early neointima formation following abdominal aortic injury. However, at 28 days following injury, no significant differences in NA or N/M ratio were detected among these three groups $(\mathrm{P}>0.05$; Fig. 5B). This loss of neointima inhibition coincided with the loss of hHGF expression at 28 days following injury.

\section{Discussion}

Neointimal hyperplasia following vascular endothelial injury may serve an important role in the pathogenesis of atherosclerosis (22) and restenosis following angioplasty (23). Endothelial injury induces the production of cytokines and growth factors by resident macrophages and VSMCs, which function via autocrine and paracrine mechanisms to stimulate and sustain VSMC proliferation and migration, eventually leading to neointima formation and restenosis of the blood vessels (1). Current therapeutic approaches to combat neointimal hyperplasia have achieved only limited success in improving clinical outcomes (24). Novel agents that prevent the early formation and progression of neointima are urgently required.

HGF was originally identified in 1984 as a mitogenic protein in rat hepatocytes (25). Later studies revealed that HGF exerts its mitogenic effects by binding to and activating the tyrosine kinase receptor, c-MET (26). HGF has been demonstrated to stimulate growth of ECs exclusively without replication of VSMCs, although its specific receptor, c-MET, is expressed in ECs and VSMCs (13). In addition to its mitogenic activity, HGF protects epithelial and endothelial cells from apoptosis under conditions of stress $(27,28)$. As a recently categorized member of the endothelium-specific growth factor family, HGF contributes to injury repair and wound healing. Previous studies on the role of hHGF in neointima formation have been somewhat controversial. Hayashi et al (16) reported that in vivo gene transfection of hHGF into the rat balloon-injured carotid artery inhibits neointima formation. In addition, intravenous infusion of HGF prevents neointimal hyperplasia in carotid expanded polytetrafluoroethylene grafting in rabbits (17). Conversely, localized treatment with a HGF fusion protein has been reported to exacerbate neointima hyperplasia in the rat balloon-injured carotid artery. This pro-neointima formation property may result from stimulation of a specific group of SMCs expressing c-MET (29,30).

In the present study, the effects of hHGF gene transfer on neointima formation in balloon-injured abdominal aortae in rabbits were investigated. Unlike previous balloon injury models, the entire injury procedure in this study was carried out under the guidance of transabdominal ultrasound with real-time tracking to ensure accurate positioning of the balloon at the target aortic segment. A consistent degree of injury was achieved by careful control of balloon inflation. Abdominal aortae of New Zealand white rabbits were selected in the present study as these vessels resemble the human coronary artery in size and structure. Arteries in smaller models including rats are too small for ultrasound monitoring. Although the porcine coronary artery model also closely simulates the human disease, the post-injury healing of porcine vessels is more rapid compared with that of humans $(29,30)$. The rabbit aortic artery exhibits a slower rate of re-endothelialization compared with the porcine model, and 
A
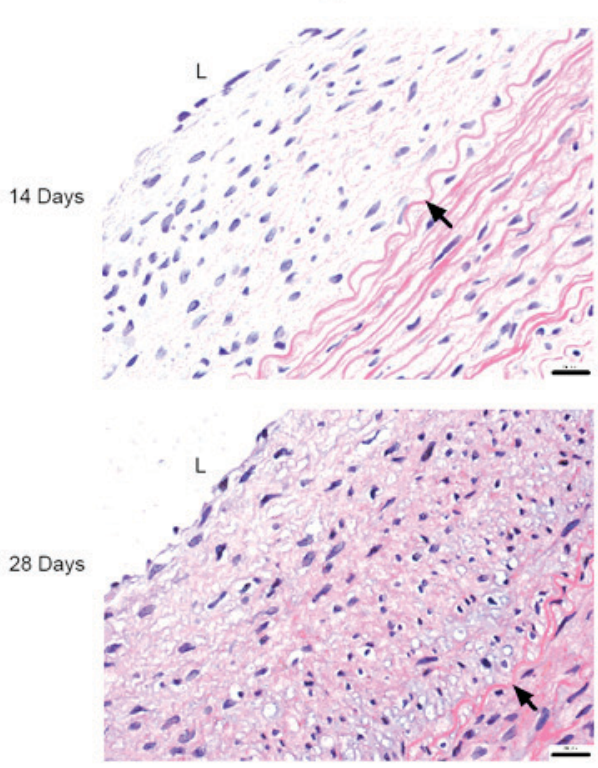

B

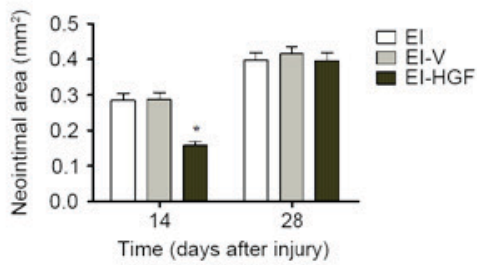

El-V
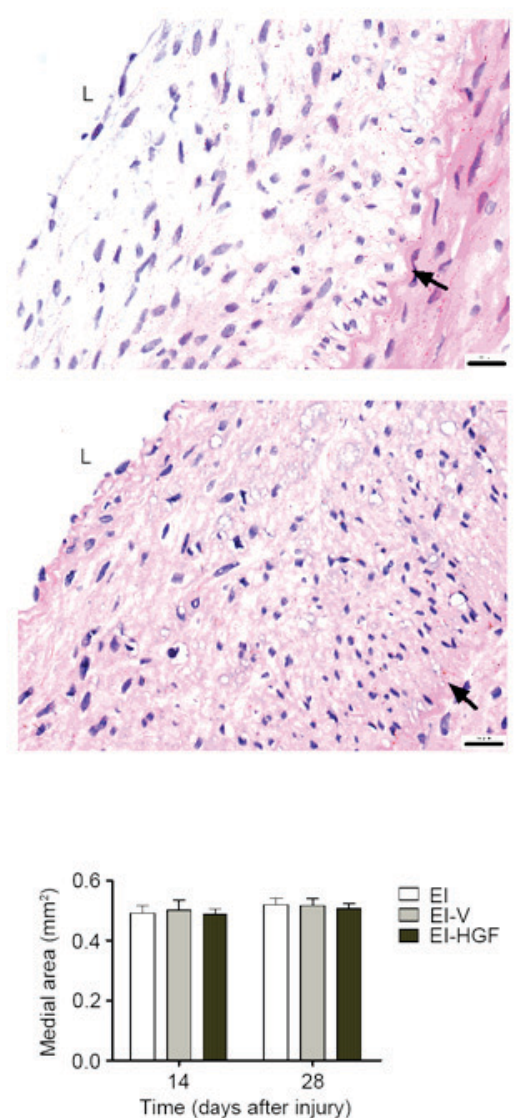
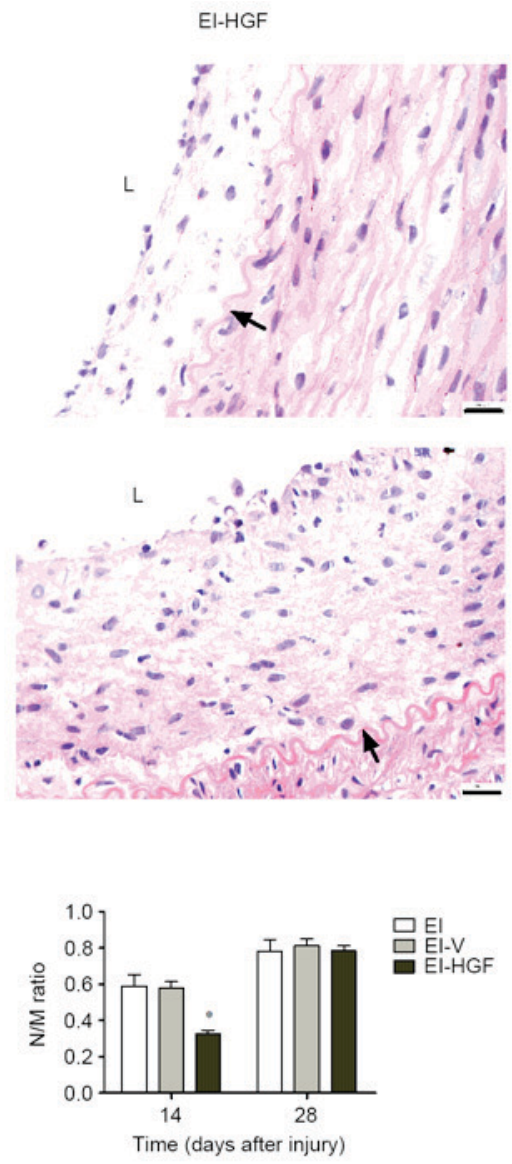

Figure 5. Effect of in vivo hHGF transfection on neointima formation at 14 and 28 days following injury. (A) H\&E staining of neointima in rabbit target aortae. Arrows indicate the internal elastic lamina, the boundary between the neointima and media. Original magnification, $\mathrm{x} 400$, scale bar, $20 \mu \mathrm{m}$. (B) Neointimal area, medial area and the N/M ratio in target aortae determined by histological analysis with H\&E staining. Values are presented as the mean \pm standard error. " P<0.05 vs. EI or EI-V on the same day. L, lumen; EI, injured aorta without transfection; EI-V, injured aorta transfected with vector alone; EI-HGF, injured aorta transfected with human hepatocyte growth factor; NA, Neointimal area; MA, medial area; N/M ratio, neointimal to medial area ratio; H\&E, hematoxylin-eosin.

thus is considered a more suitable choice. In addition, studies in preclinical restenosis models have revealed species-specific cellular and molecular mechanisms involved in vascular injury and repair (31). Specifically, rat carotid artery injury induces only a weak inflammatory response, whereas a similar injury in rabbits induces severe inflammation $(32,33)$. Taking into account all these considerations, the rabbit model was selected for the present study.

The data of the present study demonstrated decreased hHGF mRNA and protein expression levels in injured aortae compared with intact aortae, which may be attributed to impaired gene transfection and expression capacity of damaged vessels, due to a severe inflammatory response. Endothelial denudation from balloon injury rapidly induces platelet activation and aggregation followed by a thin layer thrombus formation and inflammation at the injury site (34), which may prevent effective gene transfection. During the preliminary experiments of the present study, the authors attempted to perform hHGF transfection immediately or 3 days following injury; however, both attempts were unsuccessful (data not shown). Although transfection into injured aortae was successful a week following the balloon procedure, the transfection efficiency was still repressed compared with that in intact aortae, presumably due to inflammation and early neointima formation at the injury site. This may pose a challenge in the clinical use of hHGF gene transfer for restenosis. In patients undergoing endovascular procedures, neither balloon angioplasty nor stenting resect the atherosclerotic plaque from the arteries, and by contrast, the plaque is pressed into the artery walls (35). Therefore, the underlying distorted atherosclerosis and other plaque factors, including thrombus or lipid core, may affect the efficiency of hHGF transfection.

Protective effects of hHGF transfection were observed 7 days following transfection (14 days following injury). Neointima formation in the EI-HGF group was significantly reduced compared with the EI or EI-V group. The proliferation and migration of vascular SMCs following endothelium injury serves a predominant role in neointima formation (34). The migration of SMCs into the intima disrupts EC regrowth, leading to failure in reendothelialization (36). It is possible that transfected HGF inhibits neointima formation in injured rabbit abdominal aortae by enhancing EC proliferation and reendothelialization $(17,37)$; however this would require further investigation. The protective effects of hHGF were lost 28 days following injury (21 days following transfection), which coincided with the decline in hHGF expression over time in the target vessel. Hayashi et al (16) reported that 
transfection of hHGF into rat carotid aortae immediately following injury inhibits neointima formation for up to 8 weeks. Therefore, the differences in the effects of HGF gene transfer may have resulted from differences in the animal species used in the present study (rat vs. rabbit), the target vessel (carotid vs. abdominal aortae), the degree of injury and the time of transfection. Vascular wall injuries resulting from the inflated balloon catheter vary in severity (38). The majority of balloon injuries start with deendothelialization, and may progress to deeper injury. In the present study, the inflation, positioning and movement of the balloon catheter were monitored and guided by real-time ultrasound, which ensured a consistent degree of injury. The time of transfection may also serve a primary role in determining the effects of HGF transfer. In a rabbit balloon injury model, SMCs may migrate into the neointima 5 days following injury (39). Therefore, rapid reendothelialization following injury is considered to be critical to suppress neointima formation (17). In the present study, the acute biological response to endothelium injury prevented early HGF transfection. Transfection did not occur until 7 days following the injury, which may be too late to generate lasting protective effects. Hence, the time point for transfection and the status of the vessel to be transfected may serve crucial roles in efficient and effective hHGF transfection. In the present study, hHGF gene transfection was only performed 7 days following injury with a single dosage. In future studies, it would be of interest to perform hHGF transfection prior to balloon-injury or with a number of different dosages of hHGF in order to observe its effect on arterial repair.

In conclusion, an ultrasound-guided balloon injury rabbit model was used to demonstrate that in vivo gene transfer of hHGF inhibited neointima formation in rabbit abdominal aortae for up to $\geq 2$ weeks following balloon injury. The findings of the present study suggest that HGF may be a potentially useful agent in combating neointimal hyperplasia, and the importance of early and effective HGF gene transfer remains a challenge to overcome in its clinical use for restenosis.

\section{Acknowledgements}

The present study was supported by grants from National Natural Science Foundation of China (grant nos. 31271235 and 81201094), Science and Technology Department of Jilin Province (grant no. 20130413005GH). The authors would like to thank Medjaden Bioscience Ltd. for the scientific editing of this manuscript and Beijing Northland Biotech Co., Ltd. (Beijing, China) for kindly providing the hepatocyte growth factor naked plasmid.

\section{References}

1. Libby P, Schwartz D, Brogi E, Tanaka H and Clinton SK: A cascade model for restenosis. A special case of atherosclerosis progression. Circulation 86 (6 Suppl): III47-III52, 1992.

2. Schwartz SM, Campbell GR and Campbell JH: Replication of smooth muscle cells in vascular disease. Circ Res 58: 427-444, 1986.

3. Dzau VJ and Gibbons GH: Endothelium and growth factors in vascular remodeling of hypertension. Hypertension 18 (5 Suppl): III115-III121, 1991.
4. Asahara T, Bauters C, Pastore C, Kearney M, Rossow S, Bunting S, Ferrara N, Symes JF and Isner JM: Local delivery of vascular endothelial growth factor accelerates reendothelialization and attenuates intimal hyperplasia in balloon-injured rat carotid artery. Circulation 91: 2793-2801, 1995.

5. Dulak J, Schwarzacher SP, Zwick RH, Alber H, Millonig G, Weiss C, Hügel H, Frick M, Jozkowicz A, Pachinger O and Weidinger F: Effects of local gene transfer of VEGF on neointima formation after balloon injury in hypercholesterolemic rabbits. Vasc Med 10: 285-291, 2005.

6. Simón-Yarza T, Formiga FR, Tamayo E, Pelacho B, Prosper F and Blanco-Prieto MJ: Vascular endothelial growth factor-delivery systems for cardiac repair: An overview. Theranostics 2: 541-552, 2012.

7. Johnson M, Koukoulis G, Matsumoto K, Nakamura T and Iyer A: Hepatocyte growth factor induces proliferation and morphogenesis in nonparenchymal epithelial liver cells. Hepatology 17: 1052-1061, 1993.

8. Bottaro DP, Rubin JS, Faletto DL, Chan AM, Kmiecik TE, Vande Woude GF and Aaronson SA: Identification of the hepatocyte growth factor receptor as the c-met proto-oncogene product. Science 251: 802-804, 1991.

9. Matsumoto K and Nakamura T: Emerging multipotent aspects of hepatocyte growth factor. J Biochem 119: 591-600, 1996.

10. Ponzetto C, Bardelli A, Zhen Z, Maina F, dalla Zonca P, Giordano S, Graziani A, Panayotou G and Comoglio PM: A multifunctional docking site mediates signaling and transformation by the hepatocyte growth factor/scatter factor receptor family. Cell 77: 261-271, 1994.

11. Rosen EM, Nigam SK and Goldberg ID: Scatter factor and the c-met receptor: A paradigm for mesenchymal/epithelial interaction. J Cell Biol 127: 1783-1787, 1994.

12. Matsumoto K and Nakamura T: Hepatocyte growth factor (HGF) as a tissue organizer for organogenesis and regeneration. Biochem Biophys Res Commun 239: 639-644, 1997.

13. Nakamura Y,Morishita R, Higaki J, Kida I, Aoki M, Moriguchi A, Yamada K, Hayashi S, Yo Y, Matsumoto K, et al: Expression of local hepatocyte growth factor system in vascular tissues. Biochem Biophys Res Commun 215: 483-488, 1995.

14. Nakamura Y, Morishita R, Nakamura S, Aoki M, Moriguchi A, Matsumoto K, Nakamura T, Higaki J and Ogihara T: A vascular modulator, hepatocyte growth factor, is associated with systolic pressure. Hypertension 28: 409-413, 1996.

15. Nakamura Y,Morishita R, Higaki J, Kida I, Aoki M, Moriguchi A, Yamada K, Hayashi S, Yo Y, Nakano H, et al: Hepatocyte growth factor is a novel member of the endothelium-specific growth factors: Additive stimulatory effect of hepatocyte growth factor with basic fibroblast growth factor but not with vascular endothelial growth factor. J Hypertens 14: 1067-1072, 1996.

16. Hayashi K, Nakamura S, Morishita R, Moriguchi A, Aoki M, Matsumoto K, Nakamura T, Kaneda Y, Sakai N and Ogihara T: In vivo transfer of human hepatocyte growth factor gene accelerates re-endothelialization and inhibits neointimal formation after balloon injury in rat model. Gene Ther 7: 1664-1671, 2000

17. Harada M, Takenaka H, Ikenaga S, Zhang H, Zempo N, Esato K, Nagano T, Taiji M and Noguchi H: Hepatocyte growth factor prevents intimal hyperplasia in rabbit carotid expanded polytetrafluoroethylene grafting. J Vasc Surg 35: 786-791, 2002.

18. National Research Council Committee for the Update of the Guide for the $\mathrm{C}$ and Use of Laboratory A: The National Academies Collection: Reports funded by National Institutes of Health. In: Guide for the Care and Use of Laboratory Animals National Academies Press (US) National Academy of Sciences., Washington (DC), 2011.

19. Maillard L, Van Belle E, Smith RC, Le Roux A, Denéfle P, Steg G, Barry JJ, Branellec D, Isner JM and Walsh K: Percutaneous delivery of the gax gene inhibits vessel stenosis in a rabbit model of balloon angioplasty. Cardiovasc Res 35: 536-546, 1997.

20. Brasselet C, Durand E, Addad F, Al Haj Zen A, Smeets MB, Laurent-Maquin D, Bouthors S, Bellon G, de Kleijn D, Godeau G, et al: Collagen and elastin cross-linking: A mechanism of constrictive remodeling after arterial injury. Am J Physiol Heart Circ Physiol 289: H2228-H2233, 2005.

21. Chomczynski P: A reagent for the single-step simultaneous isolation of RNA, DNA and proteins from cell and tissue samples. Biotechniques 15: 532-534, 536-537, 1993.

22. Rajendran P, Rengarajan T, Thangavel J, Nishigaki $\mathrm{Y}$, Sakthisekaran D, Sethi G and Nishigaki I: The vascular endothelium and human diseases. Int J Biol Sci 9: 1057-1069, 2013. 
23. Seedial SM, Ghosh S, Saunders RS, Suwanabol PA, Shi X, Liu B and Kent KC: Local drug delivery to prevent restenosis. J Vasc Surg 57: 1403-1414, 2013

24. Collins MJ, Li X, Lv W, Yang C, Protack CD, Muto A, Jadlowiec CC, Shu C and Dardik A: Therapeutic strategies to combat neointimal hyperplasia in vascular grafts. Expert Rev Cardiovasc Ther 10: 635-647, 2012.

25. Nakamura T, Nawa K and Ichihara A: Partial purification and characterization of hepatocyte growth factor from serum of hepatectomized rats. Biochem Biophys Res Commun 122: 1450-1459, 1984.

26. Vigna E, Naldini L, Tamagnone L, Longati P, Bardelli A, Maina F, Ponzetto C and Comoglio PM: Hepatocyte growth factor and its receptor, the tyrosine kinase encoded by the c-MET proto-oncogene. Cell Mol Biol (Noisy-le-grand) 40: 597-604, 1994.

27. Yo Y, Morishita R, Nakamura S, Tomita N, Yamamoto K, Moriguchi A, Matsumoto K, Nakamura T, Higaki J and Ogihara T: Potential role of hepatocyte growth factor in the maintenance of renal structure: Anti-apoptotic action of HGF on epithelial cells. Kidney Int 54: 1128-1138, 1998.

28. Yamamoto K, Morishita R, Hayashi S, Matsushita H, Nakagami H, Moriguchi A, Matsumoto K, Nakamura T, Kaneda Y and Ogihara T: Contribution of Bcl-2, but not Bcl-xL and Bax, to antiapoptotic actions of hepatocyte growth factor in hypoxia-conditioned human endothelial cells. Hypertension 37: 1341-1348, 2001

29. Ohkawara N, Ueda H, Shinozaki S, Kitajima T, Ito Y, Asaoka H, Kawakami A, Kaneko E and Shimokado K: Hepatocyte growth factor fusion protein having collagen-binding activity (CBD-HGF) accelerates re-endothelialization and intimal hyperplasia in balloon-injured rat carotid artery. J Atheroscler Thromb 14: 185-191, 2007.
30. Aoyagi M, Yamamoto S, Azuma H, Yamamoto M, Tamaki M, Niimi Y, Hirakawa K and Yamamoto K: Localization and effects of hepatocyte growth factor on smooth muscle cells during neointimal formation after balloon denudation. Histochem Cell Biol 111: 419-428, 1999.

31. Touchard AG and Schwartz RS: Preclinical restenosis models: Challenges and successes. Toxicol Pathol 34: 11-18, 2006.

32. Schwartz RS, Holmes DR Jr and Topol EJ: The restenosis paradigm revisited: An alternative proposal for cellular mechanisms. J Am Coll Cardiol 20: 1284-1293, 1992.

33. Miyauchi K, Aikawa M, Tani T, Nakahara K, Kawai S, Nagai R, Okada R and Yamaguchi H: Effect of probucol on smooth muscle cell proliferation and dedifferentiation after vascular injury in rabbits: Possible role of PDGF. Cardiovasc Drugs Ther 12: 251-260, 1998

34. Chaabane C, Otsuka F, Virmani R and Bochaton-Piallat ML: Biological responses in stented arteries. Cardiovasc Res 99: 353-363, 2013.

35. Schwartz RS, Chronos NA and Virmani R: Preclinical restenosis models and drug-eluting stents: Still important, still much to learn. J Am Coll Cardiol 44: 1373-1385, 2004.

36. Casscells W: Migration of smooth muscle and endothelial cells. Critical events in restenosis. Circulation 86: 723-729, 1992.

37. Chang H, Ren KF, Wang JL, Zhang H, Wang BL, Zheng SM, Zhou YY and Ji J: Surface-mediated functional gene delivery: An effective strategy for enhancing competitiveness of endothelial cells over smooth muscle cells. Biomaterials 34: 3345-3354, 2013.

38. Clowes AW and Clowes MM: Kinetics of cellular proliferation after arterial injury. IV. Heparin inhibits rat smooth muscle mitogenesis and migration. Circ Res 58: 839-845, 1986.

39. Davies MG and Hagen PO: Pathobiology of intimal hyperplasia. Br J Surg 81: 1254-1269, 1994 